\title{
Editorial
}

\author{
Volume 14 Number 2, December 2015
}

\section{Team Approach in the Management of Diabetic Foot: The Way Forward}

The incidence and prevalence of diabetes mellitus is increasing worldwide. World Health Organization (WHO) estimates that more than 350 million people worldwide have diabetes mellitus. According to the Malaysian National Health Morbidity Survey (NHMS) IV 2011, the prevalence of diabetes mellitus for both known and undiagnosed cases increased from $11.6 \%$ in 2006 to $15.2 \%$ in $2011 .{ }^{1}$ Prevalence in Malaysia is in the list of top 10 countries for diabetes in the world with the current diabetes population of approximately 3 million people. ${ }^{2}$ The warning by the WHO that diabetes is on the rise in developing countries make the primary prevention of diabetes mellitus a crucial task. Many believe that it is a disease that can be prevented by appropriate interventions in high risk individuals.

One of the major complications of diabetes mellitus is the 'diabetic foot'. It is characterized by ulceration and infection leading to amputations that contribute to high morbidity and mortality among these patients. These complications result from a complex interplay of ischemia, neuropathy, ulceration and infection. About $15 \%$ of patients suffering from diabetes mellitus will develop a lower extremity ulcer during the course of their disease. It is estimated that $70 \%$ of all nontraumatic amputations are carried out in diabetic patients.

Major amputation rate is 15 times greater than that of the general population. In addition, a diabetic patient with one lower limb amputation, has a $50 \%$ chance of developing a serious lesion in the second limb within 2 years. ${ }^{3}$ Foot problems remain the commonest cause of hospital admission amongst diabetic patients. Hospital stay is frequently prolonged and many of the patients have a history of a previous foot ulcer.

High-risk diabetic foot patient is always in danger. Salvage of the foot requires rapid recognition and appropriate attempts to save the foot and patient's life. People with neuropathic or neuro-ischaemic feet can die quickly from a cascade of events initiated by their foot problem. Recognizing an ulcer early and relieving the effects of high pressure and infection can lead to rapid healing without scarring and avoid a lifetime of disability. They can be reduced through appropriate prevention and management. Proper screening program is important to identify foot at risk or early abnormalities so that proper interventions or measures can be initiated to prevent the progression of the disease. ${ }^{4}$ The social consequences and psychological impact of amputation resulting from reduced mobility, and increasing dependence on relatives or social services is equally important and need to be handled by correct personnel.

Diabetic foot has been a neglected area for a long period. Management was shared by specialties of medicine, orthopaedic and vascular surgery in an uncoordinated approach. Multi-disciplinary teams are essential for optimal management of the high-risk diabetic foot and there is no room for inter-professional rivalry. Development of considerable mutual respect between team members from different specialties is essential for a successful outcome.

The aim in management of diabetic foot is to identify the patient at as an early stage of disease. It is necessary to take control of the foot early to prevent further progression. The cornerstone of this approach is to encourage early presentation to allow early diagnosis and early intervention within the multidisciplinary diabetic foot clinic. ${ }^{5}$ Multi-disciplinary team must works closely together, within the ambit of a diabetic foot clinic, which ideally is must be situated in a hospital. The team should meet regularly for joint consultations including ward rounds, radiological conferences and clinical sessions. Some roles of team members may overlap, depending on local expertise and interest.

It is extremely important to establish diabetic foot care services in a defined geographical area. ${ }^{2}$ This will be vital for patients who know where exactly to come for their routine and emergency appointments, and for local healthcare providers seek help and obtain advice and education.

Successful management of diabetic foot problem needs the expertise of a multi-disciplinary team. It is a globally accepted approach that has reduced the rate of amputation, shorten the hospital stay, cost effective and more importantly it has improved the quality of life of the patients.

\section{REFERENCES}

1. Institute for Public Health (IPH) 2011. National Health and Morbidity Survey 2011 (NHMS 2011). Vol. II: Non- Communicable Diseases; 2011: 188 pages

2. Shaw JE, Sicree RA, Zimmet PZ. Global estimates of the prevalence of Diabetes for 2010 and 2030. Diabetes Research and Clinical Practice. 2010.87: 4-14. 
3. Clinical Practice Guideline, Management of Diabetic Foot, Ministry of Malaysia, 2004 Aug.

4. Nather A, Chionh SB, Tay PL, et al. Foot screening for diabetics. Ann Acad Med Singapore 2010; 39: 472-5.

5. Edmonds ME, Foster AVM, Lee J. A practical manual of diabetic foot care. Sanders. 2nd ed. 2008

Assoc. Prof. Dr. Aminudin Che Ahmad

Department of Orthopaedics, Traumatology \& Rehabilitation

Kulliyyah of Medicine

International Islamic University Malaysia 